\title{
Die Entscheidung über den ambulanten Arzttarif liegt bei Ihnen
}

\section{Christian Oeschger}

Wissenschaftlicher Mitarbeiter, Abteilung Ambulante Tarife und Verträge Schweiz

\author{
Am 28. April 2016 hat die Ärztekammer der FMH eine Urabstimmung angeordnet, \\ um die Stellungnahme der ordentlichen FMH-Mitglieder zum revidierten ambu- \\ lanten Tarif einzuholen. Nun sind Sie gefragt: Sie dürfen den neuen Tarif beurtei- \\ len und müssen Ihren Stimmzettel bis spätestens am 28. Mai 2016 per Post dem auf \\ dem Couvert aufgeführten Notariat einsenden.
}

In den vergangenen vier Jahren haben sich rund 200 Tarifdelegierte der Fachgesellschaften der FMH zusammen mit den Tarifexperten der Revisionspartner H+ und MTK intensiv mit der Gesamtrevision des ambulanten Arzttarifs auseinandergesetzt. Sie alle haben dabei mit viel Herzblut an unendlich vielen Sitzungen die anfänglich mehr als 4500 Tarifpositionen überarbeitet. Die überarbeitete Struktur haben wir Ihnen Ende März 2016 über die Plattform myFMH zugänglich gemacht. Am 28. April 2016 hat die Ärztekammer beschlossen, die

Sie als Ärztin oder Arzt sollen jene Leistungen abrechnen können, die Sie auch erbracht haben.

Tarifstruktur mittels einer Urabstimmung unter allen FMH-Mitgliedern genehmigen zu lassen. Wir möchten die Gelegenheit nutzen, Ihnen nochmals ausführlich aufzuzeigen, was in den vergangenen Monaten am Tarif verändert wurde.

\section{Aktualisierung der Spartenkalkulationen}

Obwohl die Berechnungsgrundlagen bereits bei der Einführung des TARMED im Jahre 2004 mehrere Jahre alt waren, werden sie noch heute unverändert angewandt. In der Zwischenzeit hat sich auf der Kostenseite erwiesenermassen einiges getan. Während die Anlagenutzungskosten vor allem im Bereich der Geräte und Apparate gesunken sind, haben die Personalkosten deutlich zugenommen. Auf den Zahlen der Rollenden Kostenstudie RoKo basierende Schätzungen der Ärztekasse und der FMH gehen davon aus, dass in den vergangenen Jahren die Gesamtkosten um beinahe 30 Prozent angestiegen sind. Ein wichtiges Ziel der Revision war es, diese Kosten neu zu kalkulieren und aktualisiert bei den Leistungen zu hinterlegen.
Die Tarifexperten haben dazu die drei zur Anwendung kommenden Kostenmodelle "Ärztliche Leistung", «KOREG» und «INFRA» mit aktualisierten Daten gefüttert und für jede Leistung die Taxpunkte für die ärztliche Leistung (AL) sowie für die Infrastruktur- und Personalleistung (IPL, früher technische Leistung TL) errechnet. In der AL wurde zur Festlegung des wichtigsten Parameters, des Referenzeinkommens, das sogenannte Opportunitätsprinzip herangezogen. Hierbei wird unterstellt, dass ein freipraktizierender Arzt* über seine gesamte Lebensarbeitszeit ein Einkommen erzielt, das jenem eines Arztes mit festem Anstellungsverhältnis in einem Spital entspricht. Dieser Referenzwert wird wiederum durch die Jahresarbeitszeit in Minuten geteilt. Die Jahresarbeitszeit berücksichtigt sowohl Wochenenden und Feiertage als auch Absenzen diverser Art wie Ferien, Schwangerschaft oder Krankheit sowie die Fortbildung. Für das INFRAModell wurden zur Berechnung der IPL-Kostensätze unter anderem sämtliche Geräte und Apparate sowie die gesamten Parameter im Bereich der Personalkosten aktualisiert. Und auch das KOREG-Modell haben wir mit aktuellen Werten aus der RoKo gefüttert und somit die Kostenentwicklung der vergangenen Jahre einfliessen lassen.

\section{Urs Stoffel zu den Alternativen der Tarifrevision}

Für Urs Stoffel, Mitglied des Zentralvorstandes der FMH und Verantwortlicher des Departementes Ambulante Tarife und Verträge Schweiz, ist klar, dass es nicht viele Alternativen gibt: "Der Bundesrat hat in mehreren Stellungnahmen und in Gesprächen immer wieder klar festgehalten, dass er von den Tarifpartnern eine Einreichung per 30. Juni 2016 verlangt und ansonsten einen weiteren Tarifeingriff vornehmen wird.» 
Revision Ambulanter Arzttarif -

\section{Dokumentation}

Ausführliche Informationen zum revidierten Arzttarif finden Sie auf der Online-Plattform myFMH unter folgendem Link: https://myfmh.fmh.ch $\rightarrow$ Revision Ambulanter Tarif - Dokumentation.

\section{Überarbeitung der Nomenklatur}

Revidierte Kostenmodelle gehen mit der überfälligen Anpassung der darüberliegenden Nomenklatur einher. Da die Revision der aktuellen Struktur aufgrund der Uneinigkeit zwischen den Tarifpartnern in den letzten Jahren blockiert war, gibt es auch hier grossen Nachholbedarf. Es gibt heute Leistungen, die nur über Analogiepositionen den Kostenträgern in Rechnung gestellt werden, oder den umgekehrten Fall von Leistungen, die mit dem Fortschritt in der Medizin nicht mehr zur Anwendung kommen.

Trotz der Tarifierung von neuen Leistungen in vielen Bereichen konnte die Anzahl von heute gut 4500 auf unter 2700 Positionen reduziert werden. Weiter wurden auch die Tarifmechanik und die Regeln radikal vereinfacht. Aus Sicht der Revisionspartner ergeben die heute geltenden Limitationen aus medizinischer

\section{Die Kostenentwicklung muss im Sinne der Kostenwahrheit bei den Verhandlungen des Taxpunktwertes berücksichtigt werden.}

Sicht keinen Sinn und schützen kaum vor Tarifmissbrauch - sie wurden deshalb fallengelassen. Die Partner vereinfachten ebenfalls die Kumulationsverbote: Sie als Arzt sollen jene Leistungen abrechnen können, die Sie auch erbracht haben. Für die Revisionspartner ist unbestritten, dass gemäss Art. 32 Abs. 1 KVG alle erbrachten Leistungen wirksam, zweckmässig und wirtschaftlich sein müssen.

\section{Transcodierung und Normierung}

Bereits im August 2014 hat das Bundesamt für Gesundheit (BAG) die Tarifpartner darauf hingewiesen, dass der Bundesrat einzig eine kostenneutrale Revision des Tarifs genehmigen wird (Art. 59 c KVV). Wortwörtlich hat das BAG damals festgehalten: «Wenn im Sinne des Wirtschaftlichkeitsgebotes des KVG vorgeschrieben wird, dass ein Modellwechsel bei gleichen Gegebenheiten zu keinen Mehrkosten führen darf, so ist dieses Prinzip bei einer Tarifrevision, die weniger weit als ein grundsätzlicher Wechsel des Tarifmodells gehen würde, ebenfalls zur Anwendung zu bringen.»
Neben dieser eher statischen Kostenneutralität verlangt das BAG klar auch eine sogenannte dynamische Kostenneutralität. Das BAG forderte die Tarifpartner auf, in einem Konzept mögliche Konsequenzen und Massnahmen festzuhalten, sollte sich im Verlauf der Zeit herausstellen, dass die neue Tarifstruktur eine stärkere Entwicklung des gesamten Leistungsvolumens zur Folge hat. In eine ähnliche Richtung gehen die Forderungen der Kostenträger.

Angesichts des Anstiegs des Taxpunktvolumens akzeptiert die FMH zwar eine statische Kostenneutralität auf der Strukturebene bzw. beim Einführungs-Taxpunktvolumen, nicht aber auf der Preisebene. Die Kostenentwicklung muss im Sinne der Kostenwahrheit bei den Verhandlungen des Taxpunktwertes berücksichtigt werden. Gemeinsam haben die Partner deshalb eine sogenannte Normierung der überarbeiteten Tarifstruktur vereinbart.

\section{Zwischenfazit: Was haben wir erreicht?}

Ihre Kolleginnen und Kollegen haben viel erreicht! Durch die Vereinfachung der Struktur sollte diese in Zukunft für Sie besser überblickbar und damit nach einer Eingewöhnungszeit auch besser anwendbar sein Sie können neu sämtliche ambulant erbrachten Leistungen abrechnen und sind nicht mehr angewiesen

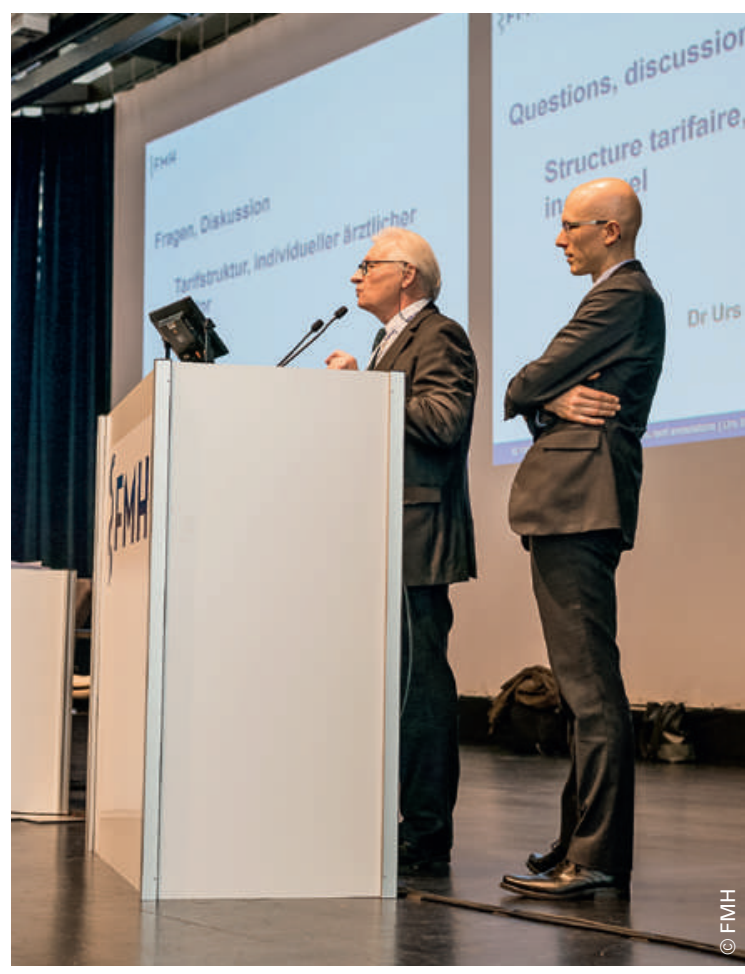

An der Ärztekammer beantworten Dr. med. Urs Stoffel, Zentralvorstand $\mathrm{FMH}$, und Patrick Müller, Abteilungsleiter Ambulante Tarife und Verträge Schweiz, die Fragen der Delegierten. 


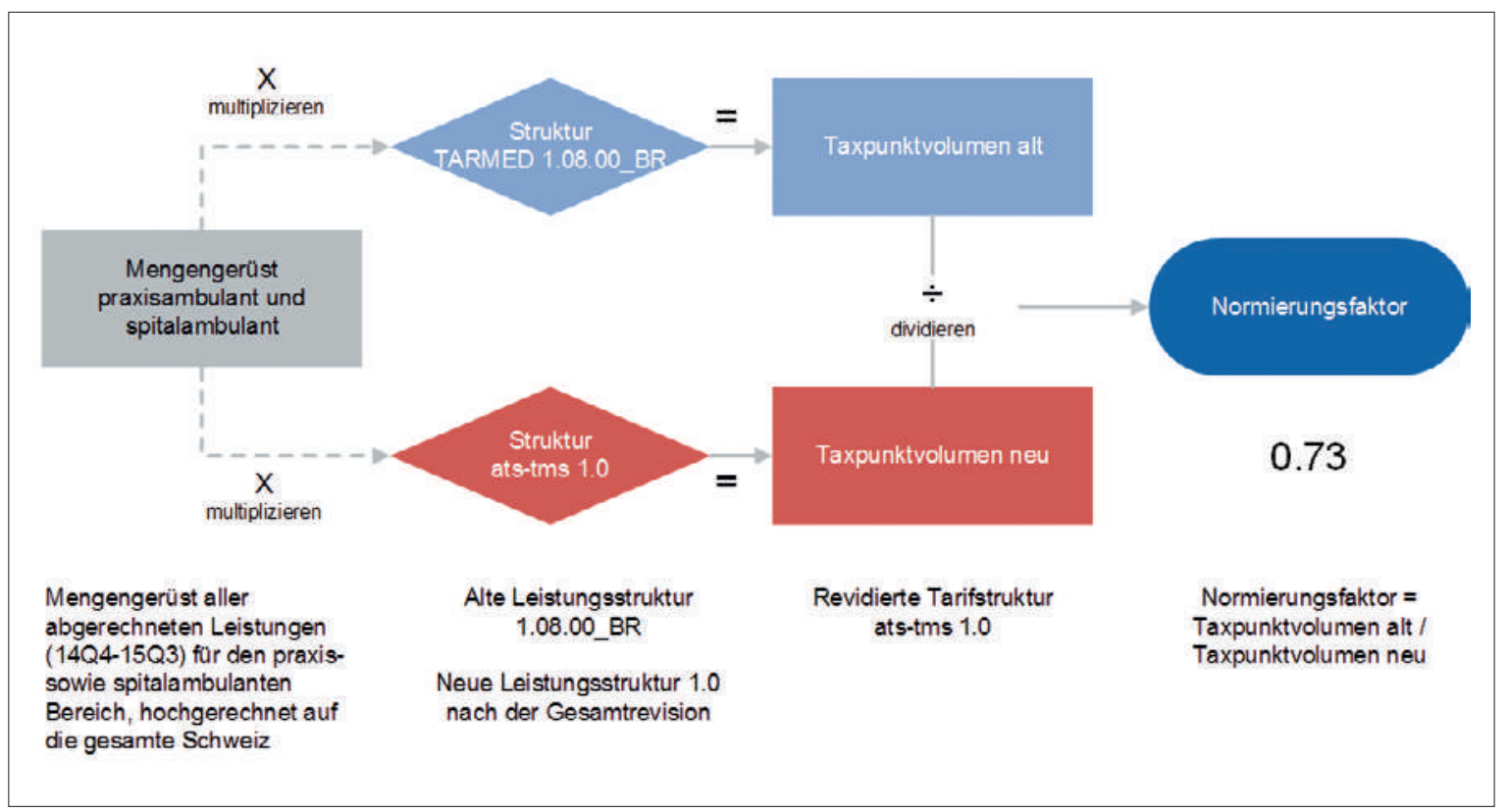

Abbildung 1: Ohne die Normierung der Tarifstruktur müsste mit einer Kostensteigerung von 37\% gerechnet werden. (Grafik: zVg vom Autor)

auf die Analogieverrechnung. Des Weiteren sind die erbrachten Leistungen nach betriebswirtschaftlichen Grundsätzen berechnet und aktualisiert worden und

\section{Die Wechselzeit wird in der aktualisierten} Tarifstruktur mittels separater Tarifpositionen abgebildet.

entsprechen den heutigen Gegebenheiten. Die neue Organisationsstruktur der ats-tms AG ermöglicht es zudem, zukünftig schneller auf die sich ändernden Rahmenbedingungen reagieren zu können.

Nichtsdestotrotz gibt es einige Neuerungen, die Sie als Arzt bei der Verrechnung der erbrachten Leistungen berücksichtigen sollten.

\section{Generelle Änderungen im neuen Tarif}

Damit es zwischen dem aktuell gültigen Tarif TARMED 1.08.00_BR und dem Tarif ats-tms 1.0 zu keinen Verwechslungen kommt, wurde bei den einzelnen Tarifpositionen von einer rein numerischen Bezeichnung auf eine alpha-numerische Nummerierung gewechselt.

\section{Tarifanwendung an konkreten Beispielen}

\section{Ärztliche und nichtärztliche Grundleistungen}

Zahlreiche Rückmeldungen von medizinischen Gesellschaften und auch von Seiten der Kostenträger haben die Revisionspartner dazu veranlasst, die anfänglich rigorose Vereinfachung im Kapitel der ärztlichen Grundleistungen nochmals zu überarbeiten. Hauptkritikpunkt war vor allem die radikale Zusammenfassung, die es im Hinblick auf mögliche WZW-Verfahren nicht erlauben würde, zwischen den Ärzten und Fachgebieten genug differenzieren zu können.

Im abschliessenden Vorschlag wird weiterhin zum einen zwischen Konsultation, Besuch und Konsilium unterschieden, zum anderen gibt es den Arztbericht sowie die ärztliche Grundleistung ohne direkten Kontakt mit

\section{Anstelle des Ausdrucks "Assistenz" wird neu} der Begriff «ärztliche Unterstützung» verwendet.

dem Patienten. Für zahlreiche Bereiche wurden wieder spezialisierte Behandlungs- und Beratungspositionen geschaffen, die zwar alle gleich bewertet sind (gleicher Kostensatz pro Minute), aber eine Differenzierung zwischen ärztlichen Grundleistungen erlauben. Neu gibt es somit ein Kapitel «Ärztliche spezialisierte Behandlungen und Beratungen» mit spezifischen Leistungen für Fachärzte, Schwerpunkte und Fähigkeitsausweise, die über keine eigenen Kapitel bzw. Unterkapitel verfügen. Eine wesentliche Änderung ergibt sich allerdings durch das neue Kapitel der nichtärztlichen Grundleistungen. Sie erlauben es zukünftig, nicht separat tarifierte Leistungen des Personals in Rechnung zu stellen. Die nichtärztlichen Leistungen sind allerdings nicht anwendbar für Leistungen, welche üblicherweise im Zusammen- 


\section{Urs Stoffel zur Normierung}

Ein juristisches Gutachten, das die FMH zusammen mit der Ärztekasse im Rahmen des Projekts Concerto in Auftrag gegeben hat, zeigt auf, dass gemäss Art. 59c Abs. 1c KVV eine Normierung des revidierten Tarifs auf das Taxpunkt-Volumen des alten Tarifs eigentlich nur bei einem Modellwechsel geltend gemacht werden kann. Die auf der Basis von Art. 59c geforderte Normierung des revidierten Arzttarifs stellt daher aus Sicht der FMH die vom KVG geforderte Wirtschaftlichkeit und Sachgerechtigkeit in Frage, da diese durch den Normierungsfaktor weitgehend wieder zerstört wird. Bei den Preisverhandlungen in den Kantonen muss die Vorgabe des KVG für die Sachgerechtigkeit und die Wirtschaftlichkeit eines Tarifs sicherlich mitberücksichtigt werden.

hang mit ärztlichen Leistungen durch nichtärztliches Personal erbracht werden. Diese Leistungen sind heute in der IPL der entsprechenden ärztlichen Leistung inkludiert.

In der Praxis bedeutet dies Folgendes: Die Positionen AM.0001 und AM.0002 werden immer zusammen angewendet, die AM.0001 gilt die reine Personalleistung (also die MPA) ab, die AM.0002 die reine Infrastruktur. Sie können also beispielsweise eine Überwachung von drei Stunden abrechnen (der Patient besetzt den Raum während drei Stunden), in der die MPA nur während zehn Minuten beim Patienten ist und den Rest der Zeit etwas anderes macht. Wenn Sie als Arzt während der Überwachung auch nach dem Patienten schauen, dann nehmen Sie zusätzlich noch die Position SK.0004 aus dem Kapitel AF.00 dazu. Die AM.0003 hingegen ist für nichtärztliche Leistungen in einer einfachen Infrastruktur wie z.B. Beratung, Information und Anweisung des Patienten, Blutentnahmen oder Wundbehandlung gedacht.

\section{Wechselzeit}

Die Wechselzeit definiert sich als durchschnittlicher Zeitaufwand (Minutage) für das Herrichten (Reinigung, Umrüsten usw.) der Infrastruktur (Sparte) zwischen der Behandlung von zwei Patienten. Die Wechselzeit wird in der aktualisierten Tarifstruktur mittels separater Tarifpositionen abgebildet. Die Wechselzeit darf pro Sparte und Sitzung nur einmal verrechnet werden.

\section{Vor- und Nachbereitung}

Bei der Vor- und Nachbereitung handelt es sich um die Zeit, die der Vor- und Nachbereitung der eigentlichen ärztlichen Kernleistung dient (z.B. steriles Waschen und Einkleiden bei einem chirurgischen Eingriff). Die Vor- und Nachbereitungszeiten der einzelnen Leistungen werden in der Regel in einer separaten Tarif- position abgebildet. Die dafür benötigte Zeit war bisher auf der entsprechenden Tarifposition eingerechnet und muss neu in den meisten Kapiteln separat verrechnet werden.

\section{Bericht}

Der Bericht gilt für die Dokumentation und das Erstellen des Befundberichts. Die Leistungserbringung auf der einen Seite sowie die Dokumentation und das Erstellen des Befundberichts auf der anderen erfolgen in der Regel nicht in derselben Infrastruktur (Sparte). Für die schriftliche Dokumentation der erbrachten Leistung ist oft eine einfache Infrastruktur ausreichend (Sprechzimmer). Im geltenden TARMED wird hierfür teilweise eine überdotierte Infrastruktur entschädigt. Deshalb wurde ein neuer Ansatz zur Tarifierung und Abgeltung des Zeitaufwandes für den Befundbericht definiert. Dieser wird grundsätzlich über die neue Tarifposition AF.0004 in Rechnung gestellt.

\section{Ärztliche Unterstützung}

Anstelle des Ausdrucks "Assistenz» wird neu der Begriff «ärztliche Unterstützung» verwendet. Als ärztliche Unterstützung gelten Personen, welche die Arzt-Ausbildung mit dem Staatsexamen erfolgreich abgeschlos-

\section{Der Bericht gilt für die Dokumentation und das Erstellen des Befundberichts.}

sen haben. Der Doktortitel ist keine zwingende Voraussetzung, da auch Fachärzte nicht in jedem Fall über den Doktortitel verfügen müssen. Nicht als unterstützender Arzt gelten angehende Ärzte, also Ärzte in Ausbildung (cand. med.), die zum nichtärztlichen Personal zählen. Die Anzahl unterstützender Ärzte ist tarifarisch auf zwei Personen limitiert. Diese müssen medizinisch notwendig sein sowie gegenüber den Kostenträgern belegt und begründet werden können. Sofern medizinisch notwendig, kann die ärztliche Unterstützung grundsätzlich bei allen Leistungen angewendet werden, muss im neuen Tarif aber separat über die Positionen SK.0001 und SK.0OO2 in Rechnung gestellt werden.

\section{Innovationen}

Die Positionen aus dem Kapitel ST Innovationen gelten lediglich für neue Leistungen, die in der aktuell gültigen Tarifstruktur noch nicht tarifiert sind. Bei der Verwendung dieser Positionen darf es zu keiner Kumulation mit spezifisch tarifierten Leistungen innerhalb der gleichen Sparte kommen. Die Positionen sind für die Revisionspartner eine Art Warnhinweis, dass in einem bestimmten Bereich allenfalls eine Verschiebung vom stationären in den ambulanten Bereich 
stattgefunden hat oder sich eine neue Behandlungsmethode etabliert hat. Die spezifische Abbildung dieser Leistungen im Tarif muss durch die medizinische Fachgesellschaft innerhalb von sechs Monaten nach erstmaliger Durchführung bei der Tariforganisation beantragt werden.

Im Bereich des OP- und Anästhesieprozesses ist es mit der Neutarifierung zu umfangreicheren Änderungen gekommen.

\section{OP- und Anästhesieprozess}

Im Bereich des OP- und Anästhesieprozesses ist es mit der Neutarifierung zu umfangreicheren Änderungen gekommen. So wurden unter anderem die einzelnen Leistungen anhand des Prozessablaufes besser voneinander getrennt, fehlende Leistungen ermittelt und aufgenommen und neu auch Leistungen zur Patientensicherheit bei Operationen tarifiert. Die allgemeinen Leistungen für den OP-Prozess sind einem eigenen Kapitel in der Nomenklatur zugeordnet: $\mathrm{RQ}$ «Allgemeine Leistungen Operationssaal OP».

\section{Ausblick}

Die FMH hat Ihnen zur besseren Beurteilung auf der Plattform myFMH (https//myfmh.fmh.ch $\rightarrow$ Revision Ambulanter Tarif - Dokumentation) diverse Simulationen und Vergleiche zwischen den beiden Tarifstrukturen zur Verfügung gestellt, um Ihnen Ihre Entscheidung zu erleichtern. Schauen Sie sich anhand konkreter Daten aus der Praxis oder der von den Fach- gesellschaften erstellten Warenkörbe an, wie sich der Tarif auf Sie als Arzt auswirkt, bilden Sie sich bis zum 28. Mai 2016 eine Meinung und geben Sie Ihre Stimme $\mathrm{ab!}$

Die FMH rechnet bis Anfang Juni 2016 mit dem Resultat aus der Urabstimmung. Sofern die Mehrheit der FMH-Mitglieder dem vorliegenden Arzttarif zustimmt, wird die FMH diesen per 30. Juni 2016 dem Bundesrat zur Genehmigung einreichen. Falls der Bundesrat den Tarif genehmigt, plant die FMH zusammen mit den Tarifpartnern dessen Einführung per Anfang 2018. Verständlicherweise sind Aufbau und Philosophie der neuen Struktur nicht alleine durch einen Tarifbrowser ersichtlich. Aus diesem Grund plant die FMH, zusam-

\section{Die FMH bedankt sich bei allen an diesem Mammut-Projekt beteiligten Personen für die ausnahmslos grosse Unterstützung.}

men mit ihren Partnern und in Absprache mit den jeweiligen Fachgesellschaften ab Herbst 2016 Schulungen durchzuführen.

\section{Grosses Dankeschön}

Die FMH bedankt sich bei allen an diesem MammutProjekt beteiligten Personen für die ausnahmslos grosse Unterstützung. Ohne den riesigen Einsatz der zahlreichen Tarifdelegierten und Präsidenten der medizinischen Gesellschaften wäre die Tarifrevision undenkbar gewesen. Viele von Ihnen haben Tag und Nacht gearbeitet. Vielen Dank! 